\title{
Entanglement Spectrum of Chiral Fermions on the Torus
}

\author{
Pascal Fries $\odot^{1, *}$ and Ignacio A. Reyes ${ }^{2, \dagger}$ \\ ${ }^{1}$ Fakultät für Physik und Astronomie, Julius-Maximilians Universität Würzburg, Am Hubland, 97074 Würzburg, Germany \\ ${ }^{2}$ Max-Planck-Institut für Gravitationsphysik, Am Mühlenberg 1, 14476 Potsdam, Germany
}

(Received 15 May 2019; published 19 November 2019)

\begin{abstract}
We determine the modular Hamiltonian of chiral fermions on the torus, for an arbitrary set of disjoint intervals at generic temperature. We find that, in addition to a local Unruh-like term, each point is nonlocally coupled to an infinite but discrete set of other points, even for a single interval. These accumulate near the boundaries of the intervals, where the coupling becomes increasingly redshifted. Remarkably, in the presence of a zero mode, this set of points "condenses" within the interval at low temperatures, yielding continuous nonlocality.
\end{abstract}

DOI: 10.1103/PhysRevLett.123.211603

Introduction.-Amongst the predictions stemming from the interplay between quantum field theory (QFT) and the causal structure of spacetime, one of the most robust is the celebrated Unruh effect: An accelerated observer in the vacuum measures a thermal bath, with a temperature proportional to its proper acceleration [1-3]. Intimately connected with the thermodynamics of black holes via Hawking radiation, this lies at the heart of our current understanding of the quantum nature of gravity [4]. Therefore, it is natural to explore its generalizations and investigate it further.

In recent years, these phenomena have been extended into the framework of quantum information theory. There, this temperature is understood as arising from the entanglement structure of the vacuum. Starting from a state $\rho$ and some entangling subregion $V$, one defines the reduced density matrix $\rho_{V}$ by tracing out the complement of $V$. Then, just as the entanglement entropy $S_{V}=-\operatorname{Tr}\left[\rho_{V} \log \rho_{V}\right]$ generalizes the thermal entropy, the usual Hamiltonian is an instance of the more general concept of a modular (or entanglement) Hamiltonian $\mathcal{K}_{V}$ defined via

$$
\rho_{V}:=\frac{e^{-\mathcal{K}_{V}}}{\operatorname{tr} e^{-\mathcal{K}_{V}}}
$$

Originally introduced within algebraic QFT [5], the modular Hamiltonian has aroused much interest across a wide community due its close connection to quantum information measures. In the context of many body quantum systems, the spectrum of this operator is known

Published by the American Physical Society under the terms of the Creative Commons Attribution 4.0 International license. Further distribution of this work must maintain attribution to the author(s) and the published article's title, journal citation, and DOI. Funded by SCOAP ${ }^{3}$. as the "entanglement spectrum" and has been proposed as a fingerprint of topological order [6-8] and investigated in lattice models [9-13], as well as tensor networks [14-16]. In QFT, it is fundamental for the study of relative entropy $[17,18]$ and its many applications to energy and information inequalities [19-21]. In the context of the AdS/CFT correspondence, it is instrumental in the program of reconstructing a gravitational bulk from the holographic data [22-31].

However, the modular Hamiltonian is known in only a handful of cases. The result is universal and local for the vacuum of any QFT reduced to Rindler space $[3,32]$ and hence any CFT vacuum on the plane reduced to a ball [22]. For any $\mathrm{CFT}_{2}$, the same applies for a single interval, for the vacuum on the cylinder or a thermal state on the real line $[33,34]$. More generically, modular flows can be nonlocal, as is the case for multiple intervals in the vacuum of chiral fermions on the plane or the cylinder $[35,36]$ and scalars on the plane [37]. The exact nature of the transit from locality to nonlocality, however, is not fully understood, and remains an active topic of research.

In this Letter we report progress regarding this problem, by providing a new entry to this list. We show that the chiral fermion on the torus (finite temperature on the circle) is a solvable model that undergoes such a transition between locality and nonlocality. We compute the modular Hamiltonian by restating the problem as a singular integral equation, which in turn we solve via residue analysis.

Let us quickly quote our main result. For generic temperature, the modular Hamiltonian takes the form

$$
\mathcal{K}_{\text {loc }}+\mathcal{K}_{\text {biloc }}
$$

The local flow is of the standard Rindler form (7), with entanglement temperature given in (19). The novel result is the second term, given in (23) and depicted in Fig. 2, involving bilocal couplings between a discrete but infinite 
set of other points within the subregion. In the low temperature limit, the sector with a zero mode experiences a "condensation" of these points, resulting in a completely nonlocal flow.

The resolvent.-We start by introducing the resolvent method, following $[35,37,38]$. For any spatial region $V$, the reduced density matrix $\rho_{V}$ is defined as to reproduce expectation values of local observables supported on $V$. Now, for free fermions, Wick's theorem implies that it is sufficient that $\rho_{V}$ reproduces the equal-time Green's function

$$
\operatorname{Tr}\left[\rho_{V} \psi(x) \psi^{\dagger}(y)\right]=\left\langle\psi(x) \psi^{\dagger}(y)\right\rangle=: G(x, y)
$$

for $x, y \in V$. This requirement fixes the modular Hamiltonian to be a quadratic operator given by [39]

$$
\mathcal{K}_{V}=\int_{V} d x \int_{V} d y K_{V}(x, y) \psi^{\dagger}(x) \psi(y)
$$

with kernel $K_{V}=-\log \left[\left.G\right|_{V} ^{-1}-1\right]$. This is specific for the free fermion. $G_{V}$ refers to the propagator as the kernel of an operator acting on functions with support on $V$.

As shown in [35] the modular Hamiltonian can be rewritten as

$$
K_{V}=-\int_{1 / 2}^{\infty} d \xi\left[R_{V}(\xi)+R_{V}(-\xi)\right]
$$

in terms of the resolvent of the propagator,

$$
R_{V}(\xi):=\left(\left.G\right|_{V}+\xi-1 / 2\right)^{-1} .
$$

A derivation of (3) is provided in the Supplemental Material [40]. In essence, it is the operator version of

$$
\log X=\frac{1}{2 \pi i} \oint_{\gamma} d z \frac{\log z}{z-X}
$$

for a suitable choice of contour $\gamma$.

In (4), the inverse of an operator is understood in the sense of a kernel,

$$
\int_{V} d z R_{V}(\xi ; x, z)[G(z, y)+(\xi-1 / 2) \delta(z, y)]=\delta(x-y) .
$$

Thus, provided $G$ of the global state and the entangling region $V$, this equation completely determines the resolvent $R_{V}$ and hence the modular Hamiltonian via (3).

To obtain the resolvent, let us first do the redefinition

$$
R_{V}(\xi ; x, y)=\frac{\delta(x-y)}{\xi-1 / 2}-\frac{F_{V}(\xi ; x, y)}{(\xi-1 / 2)^{2}}
$$

The convenience of this is that the first term of (5) will cancel the right-hand side of the previous equation, translating (4) into a singular integral equation

$$
\begin{aligned}
0= & G(x, y)-F_{V}(\xi ; x, y) \\
& -\frac{1}{\xi-1 / 2} \int_{V} d z G(x, z) F_{V}(\xi ; z, y) .
\end{aligned}
$$

All previous considerations hold for free fermions on a generic Riemann surface. The simplest case is the plane where the solution of (6) is a standard result [41], which was used by [35] to derive the corresponding modular Hamiltonian. They found that for multiple intervals, it consists of a local and a bilocal term. The former can be written as

$$
\mathcal{K}=\int_{V} d x \beta(x) T(x)
$$

in terms of the stress tensor $T=(\mathrm{i} / 2)\left[\psi^{\dagger} \partial_{x} \psi-\psi \partial_{x} \psi^{\dagger}\right]$, where $\beta(x)$ is known as the entanglement temperature. On the other hand, the bilocal term couples the field between different intervals.

Let us now proceed to the case of a chiral fermion on the torus. As is customary, we take the periods to be $1, \tau$ with $\Im(\tau)>0$, such that the nome $q:=e^{i \pi \tau}$ is inside the unit disk. We restrict our discussion to purely imaginary modulus $\tau=i \beta$, where $\beta$ is the inverse temperature-the general case can be recovered by analytic continuation. For simplicity, we move to radial coordinates $w=e^{i \pi z}$.

Since we are dealing with fermions, the correlator $G(u, v)$ with $u=e^{i \pi x}$ and $v=e^{i \pi y}$ is either periodic (Ramond; R) or antiperiodic (Neveu-Schwarz; NS) with respect to either of the two periods of the torus. We focus on the "thermal" case, with NS periodicity with respect to $\tau$. Combining this with the requirement to reproduce the UV correlator $G^{\mathrm{UV}}(x, y)=[2 \pi i(x-y)]^{-1}$ on small scales fully determines the standard Green's functions [42]

$$
G^{\nu}(u, v)=\frac{\eta^{3}\left(q^{2}\right)}{i \vartheta_{1}\left(u v^{-1} e^{\epsilon} \mid q\right)} \frac{\vartheta_{\nu}\left(u v^{-1} \mid q\right)}{\vartheta_{\nu}(1 \mid q)},
$$

where $\eta(q)$ and $\vartheta_{\nu}(z \mid q)$ are the Dedekind eta and Jacobi theta functions (see Supplemental Material [40]).

Here, the superscript

$$
\nu=2,3=(\mathrm{R}, \mathrm{NS}),(\mathrm{NS}, \mathrm{NS})
$$

labels the different spin structures, and we introduced a regulator $\epsilon$ in order to treat the distribution $G^{\nu}$ as a function. The sign of $\epsilon$ depends on the chirality-without loss of generality, we choose $\epsilon>0$.

With the notation settled, we now go back to the integral equation (6). In radial coordinates, it reads

$$
\begin{aligned}
0= & G^{\nu}(u, v)-F_{V}^{\nu}(\xi ; u, v) \\
& -\frac{1}{\xi-1 / 2} \frac{1}{i \pi} \int_{A} \frac{\mathrm{d} w}{w} G^{\nu}(u, w) F_{V}^{\nu}(\xi ; w, v)
\end{aligned}
$$




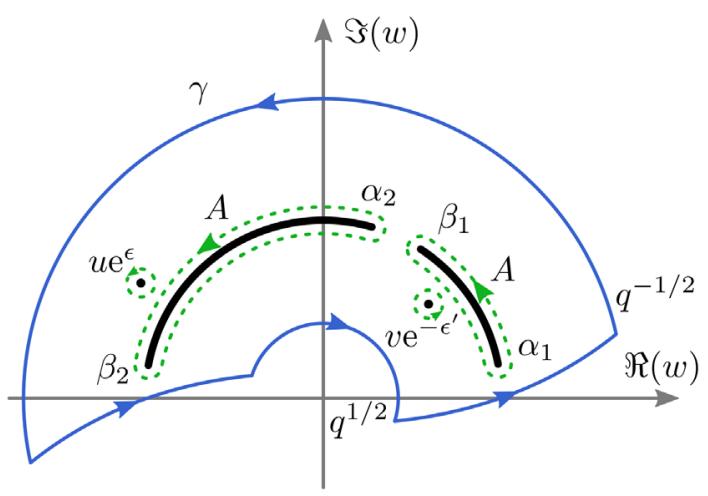

FIG. 1. The complex plane analysis in the argument. The black solid line is the entangling region-here for simplicity two intervals. The blue line represents the contour of integration $\gamma$ in (10), which leads to the residues evaluated along the green dotted curves.

with $A:=e^{i \pi V}$ being the entangling region. The key observation of this Letter is that (9) resembles the result of a contour integral, involving simple poles and branch cuts. Thus the strategy to solving (9) is to recast it as a contour integral.

To this end, we start by listing a set of sufficient properties that $F_{V}^{\nu}$ must possess in order to solve this equation:

(A) Periodicities. First, it must have the same periodicities in the $w$ argument as $G^{\nu}$, such that $G^{\nu} F_{V}^{\nu}$ is well defined on the torus. The reason is that doubly periodic functions have vanishing residue along the boundary $\gamma$ of any fundamental region (see Fig. 1):

$$
0=\frac{1}{i \pi} \oint_{\gamma} \frac{\mathrm{d} w}{w} G^{\nu}(u, w) F_{V}^{\nu}(\xi ; w, v) .
$$

Our aim is now to rewrite this in the form of (9).

(B) Location of poles and branch cuts. The next property we demand is that $F_{V}^{\nu}$ have a simple pole $F_{V}^{\nu}(u, v) \sim$ $1 / 2\left(u v^{-1}-1\right)$ at $u \rightarrow v$, together with a branch cut along the entangling region $A$, which we specify below. Everywhere else it must be analytic. Note that, similarly to $G^{\nu}$, we need to introduce a regulator $\epsilon^{\prime}>0$ for the pole of $F_{V}^{\nu}$.

If these conditions are met, a simple residue analysis shows that (10) reduces to

$$
\begin{aligned}
0= & G^{\nu}\left(u, v e^{-\epsilon^{\prime}}\right)-F_{V}^{\nu}\left(\xi ; u e^{\epsilon}, v e^{-\epsilon^{\prime}}\right) \\
& -\frac{1}{\xi-1 / 2} \frac{1}{i \pi} \int_{A^{\circlearrowleft}} \frac{\mathrm{d} w}{w} G^{\nu}\left(u e^{\epsilon}, w\right) F_{V}^{\nu}\left(\xi ; w, v e^{-\epsilon^{\prime}}\right),
\end{aligned}
$$

where we made the regulators explicit and $A^{\circlearrowleft}$ denotes a snug path around the cut on $A$ as depicted in Fig. 1.

(C) Residues. This last integral decomposes into three contributions: one along $A$ just inside the unit circle, one along $A$ just outside the unit circle, and contributions from the boundary points $\alpha_{n}=e^{i \pi a_{n}}, \beta_{n}=e^{i \pi b_{n}}$ of $A$ as can be seen from Fig. 1. Our final requirements on $F_{V}^{\nu}$ are that the residues at $\partial^{\# 1} A$ vanish, while $F_{V}^{\nu}$ has to have a multiplicative branch cut along $A$ : at every point, the ratio of the function just above and below the cut is a fixed number

$$
\frac{F_{V}^{\nu}\left(u e^{-\epsilon^{\prime \prime}}, v\right)}{F_{V}^{\nu}\left(u e^{+\epsilon^{\prime \prime}}, v\right)}=\frac{\xi+1 / 2}{\xi-1 / 2}=: e^{2 \pi h}
$$

The solution to (12) in the plane is familiar: $F(z)=z^{m}$ with $m \notin \mathbb{Z}$ possesses such a cut. Below we find the analog of this on the torus.

If properties (A), (B), (C) are satisfied, it is easy to show that such an $F_{V}$ indeed solves the problem: our contour equation (11) becomes exactly the original singular integral equation (9). The requirement that the residues on $\partial A$ vanish is equivalent to demanding that the modular flow behaves like Rindler space in the vicinity of $\partial A$. This is analogous to the derivation of the black hole temperature by the smoothness condition at the horizon.

In the Supplemental Material [40], we explicitly derive $F_{V}^{\nu}$ satisfying all of the above assumptions. The general procedure is as follows: (1) Start with the standard solution for the requirement of a multiplicative branch cut (12) on the cylinder [36]. (2)Average over all fundamental domains in the direction of $\tau$. This yields a quasiperiodic function. (3) Multiply with a slightly modified form of the Green's function (8) to turn the quasiperiodicity into a periodicity and introduce the correct pole.

We are now in position to state one of the main results of this Letter: the resolvent for a finite union of disjoint intervals on the torus, $V=\cup_{n=1}^{N}\left(a_{n}, b_{n}\right)$. The exact expression lives in the complex plane, but is vastly simplified along $A$. Introducing the shorthand notation

$$
\lambda:=\left(\prod_{n=1}^{N} \frac{\alpha_{n}}{\beta_{n}}\right)^{i h}=e^{\pi h L},
$$

where $L$ is the total length of $V$, our result is

$$
\begin{aligned}
F_{V}^{\nu}(\xi ; u, v)= & \frac{\eta^{3}\left(q^{2}\right)}{i \vartheta_{1}\left(u v^{-1} e^{\epsilon^{\prime}} \mid q\right)} \frac{\vartheta_{\nu}\left(\lambda u v^{-1} \mid q\right)}{\vartheta_{\nu}(\lambda \mid q)} \\
& \times e^{-2 \pi h}\left[\frac{\Omega_{V}(u)}{\Omega_{V}(v)}\right]^{i h}
\end{aligned}
$$

with $h$ defined in (12), and

$$
\Omega_{V}(w):=-\prod_{n=1}^{N} \frac{\vartheta_{1}\left(w \alpha_{n}^{-1} \mid q\right)}{\vartheta_{1}\left(w \beta_{n}^{-1} \mid q\right)} .
$$

Some comments are in order. The term in the second line of (14) is the complex power of a quotient, which introduces the required branch cut along $A$. This function is 
quasiperiodic, acquiring a factor of $\lambda^{2}$ when translated into the next fundamental domain. The first factor resembles the propagator (8) and introduces the desired pole, as described above. Additionally, the extra factor of $\lambda$ in the argument of $\vartheta_{\nu}$ is there to precisely cancel the quasiperiodicity of the second term. This allows the product $G^{\nu} F_{V}^{\nu}$ to be exactly doubly periodic, as required.

Modular Hamiltonian.-Finally, now that we have found the resolvent $R_{V}^{\nu}$, we can go back to (3) to obtain the modular Hamiltonian $K_{V}^{\nu}$. First, note that the leading divergence of $F_{V}^{\nu}(u, v) \sim 1 / 2\left(u v^{-1} e^{\epsilon^{\prime}}-1\right)$ at $u \rightarrow v$ can be rewritten as a Cauchy principle value

$$
\frac{1}{2} \frac{1}{u v^{-1} e^{\epsilon^{\prime}}-1}=\frac{\delta(x-y)}{2}+\mathcal{P} \frac{1}{2} \frac{1}{u v^{-1}-1} .
$$

For the sake of readability, we shall keep $\mathcal{P}$ implicit for the rest of this Letter. Equation (16) implies that the $\delta$ terms from (5) drop out in (3), yielding

$$
K_{V}^{\nu}=\int_{1 / 2}^{\infty} \frac{d \xi}{(\xi-1 / 2)^{2}}\left[F_{V}^{\nu}(\xi)+F_{V}^{\nu}(-\xi)\right] .
$$

The main characteristic of (17) is that the integrand is highly oscillatory and divergent around $\xi=1 / 2$. Indeed, notice that when $\xi \rightarrow 1 / 2$ the prefactor in (17) diverges quadratically while $F(\xi)$ vanishes linearly but oscillates wildly due to the last factor in (14). However, this behavior is well understood in the theory of distributions, and in this sense the expression (17) is well defined and closely related to the Dirac delta.

In the Supplemental Material [40], we evaluate (17) analytically. Here we will simply quote the result, but the main steps in the derivation are the following: (1) Change variables to isolates all the infinite poles along the negative axis, which then lie in successive fundamental domains. (2) Regularize (17) by placing a contour that includes increasingly many poles, and express it by residues. (3) Use the quasiperiodicites of $\vartheta_{\nu}$ to bring every pole to the fundamental region, expressing (17) as a highly oscillatory function with a divergent prefactor. (4) Remove the regulator, leading to standard Dirichlet kernel representations of the periodic or antiperiodic Dirac delta.

The final expression for the modular Hamiltonian depends on the spin sector. Let us focus on the results for a single interval. Both sectors $\nu=2,3$ have a local and a bilocal term. The local term is identical in both cases and takes the form

$$
K_{\mathrm{loc}}(x, y)=\beta(x)\left[i \partial_{x}+f(x)\right] \delta(x-y),
$$

with the entanglement temperature

$$
\beta(x)=\frac{2 \pi \beta}{2 \pi+\beta \partial_{x} \log \Omega_{V}\left(e^{i \pi x}\right)},
$$

where $\Omega_{V}$ is as defined in (15) and the function $f(x)$ is fixed by requiring that $K_{\mathrm{loc}}$ is Hermitian. Note that the expression (18) is equivalent to the more familiar Rindler-like representation (7).

The bilocal term represents the central result of this Letter and shows a novel feature: In both sectors, it involves a coupling between an infinite but discrete set of points, and is given by

$$
\begin{aligned}
K_{\text {biloc }}^{ \pm}(x, y)= & \frac{i \pi}{L \sinh \pi \mu(x, y)} \\
& \times \sum_{k \in \mathbb{Z} \backslash\{0\}}( \pm 1)^{k} \delta(x-y+\beta \mu(x, y)-k),
\end{aligned}
$$

where the sign \pm corresponds to $\nu=\frac{2}{3}$. Here, we used the function

$$
\mu(x, y)=\frac{1}{2 \pi L} \log \frac{\Omega_{V}\left(e^{i \pi x}\right)}{\Omega_{V}\left(e^{i \pi y}\right)},
$$

which will play an important role in the analysis below.

Note that $K_{\text {biloc }}^{ \pm}$couples pairs $(x, y)$ which are solutions of

$$
x-y+\beta \mu(x, y)-k=0, \quad k \in \mathbb{Z} \backslash\{0\} .
$$

Because $\mu(x, y)$ is monotonic in $y$ and diverges at the end points, Eq. (22) possesses a unique solution for every $k$, as shown in Fig. 2. Solutions accumulate near the end points. In the next section, we analyze the above expressions and discuss their physical meaning. A summary of the results is presented in Table I.

Discussion.-In this Letter we computed the modular Hamiltonian of chiral fermions in a thermal state on the circle, reduced to an arbitrary set of disjoint intervals.

Our main result is that for arbitrary temperature, the modular Hamiltonian contains a local term, as well as an infinite number of bilocal contributions, even for a single interval. Let us now analyze the bilocal terms in more detail. Inserting the kernel (20) back into (2), the bilocal modular Hamiltonian reads

$$
\mathcal{K}_{\text {biloc }}^{ \pm}=\sum_{k \neq 0}( \pm 1)^{k} \int_{V} d x \alpha\left(x, x_{k}\right) \psi^{\dagger}(x) \psi\left[x_{k}(x)\right]
$$

As depicted in Fig. 2, the $x_{k}(x)$ are an infinite set of points within the interval, solutions to Eq. (22). The bilocal coupling $\alpha\left(x, x_{k}\right)$ has dimensions of energy and is given by

$$
\alpha(x, y)=\frac{i \pi}{L \sinh \pi \mu(x, y)} \frac{1}{\left|1-\beta \partial_{y} \mu(x, y)\right|} .
$$

Although determining the exact location of the $x_{k}$ is difficult, two properties are simple to extract: 


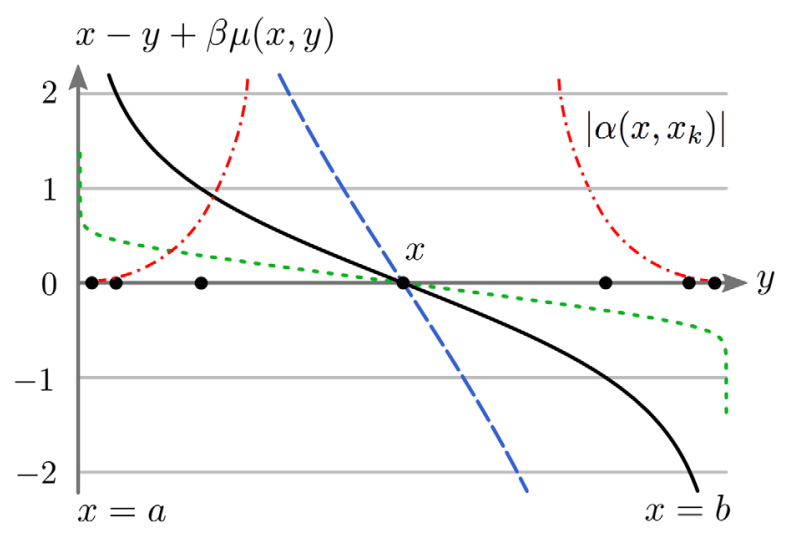

FIG. 2. For finite $\beta$ (black solid line) the point at the center is bilocally coupled to an infinite set $x_{k}(x)$ (black dots), solutions to (22) for a single interval. For large $\beta$ (blue dashed line), the solutions distribute densely, whereas for $\beta \rightarrow 0$ (green dotted line) they all localize at the end points. The strength $\alpha\left(x, x_{k}\right)$ of the coupling (red, dot-dashed line) decays towards the end points.

First, the infinite set of $x_{k}$ accumulate near the end points of the interval. Indeed, because $\mu$ diverges there, there is an infinite number of solutions near the boundaries, located at

$$
x_{k}=a+e^{-2 \pi L k / \beta}, \quad \text { as } k \rightarrow \infty
$$

and similarly near $b$.

Second, their contributions vanish as they approach the end points. Using (20), the coupling in (23) goes as

$$
\left|\alpha\left(x, x_{k}\right)\right| \stackrel{k \rightarrow \infty}{\rightarrow} \frac{4 \pi^{2}}{\beta}\left(x_{k}-a\right)^{1+1 / 2 L} .
$$

The energy scale of $\alpha\left(x, x_{k}\right)$ is set by the temperature $\beta^{-1}$, whereas the falloff is determined by the length of the interval $L$. Interestingly, the strength of the nonlocal couplings appears to be "redshifted" due to their proximity to the local Rindler horizons located at the end points.

As a next step, let us see how to recover the known results at very high [34] and low [36] temperatures. We start with the high temperature limit $\beta \rightarrow 0$. One easily sees from (19) that the local term goes as the inverse temperature, $\beta(x) \sim \beta$, as expected. On the other hand, as depicted in Fig. 2, the bilocal contributions (20) all approach the end points, where they vanish exponentially.

Moving now to the low temperature limit $\beta \rightarrow \infty$, the entanglement temperature (19) approaches the well-known result for the cylinder [36]

$$
\lim _{\beta \rightarrow \infty} \beta(x)=\frac{2 \pi}{\partial_{x} \log \frac{\sin (x-a)}{\sin (b-x)}} .
$$

The bilocal contributions, however, behave remarkably. As can be understood from Fig. 2, as we lower the temperature, the curve gets increasingly steep. Thus, the
TABLE I. Summary of our results for the modular Hamiltonian in different spin sectors. The definitions for $K_{\text {loc }}$ and $K_{\text {biloc }}^{ \pm}$are in (18)-(20). The local and nonlocal terms at low temperature $(\beta \rightarrow \infty)$ are given in (26) and (27).

\begin{tabular}{lccc}
\hline \hline$\nu$ & $\beta \rightarrow \infty$ & $\beta$ finite & $\beta \rightarrow 0$ \\
\hline 2 & Local + continuous nonlocal & $K_{\text {loc }}+K_{\text {biloc }}^{+}$ & $\beta i \partial_{x} \delta(x-y)$ \\
3 & Local & $K_{\text {loc }}+K_{\text {biloc }}^{-}$ & $\beta i \partial_{x} \delta(x-y)$ \\
\hline \hline
\end{tabular}

solutions to (22) form a partition of the interval which becomes denser and denser in the limit $\beta \rightarrow \infty$. Now, recall that the modular Hamiltonian must always be thought of as a distribution, i.e., as integrated against regular test functions. In this limiting procedure, the solutions to (22) "condense" in the interval, and it can be shown that the sequence of Dirac deltas in (20) reproduce precisely the definition of a Riemann integral. Indeed, one can show that in this sense (20) becomes completely nonlocal

$$
\lim _{\beta \rightarrow \infty} K_{\text {biloc }}^{+}(x, y)=\frac{i \pi}{L \sinh \pi \mu(x, y)},
$$

in agreement with [36], whereas $\lim _{\beta \rightarrow \infty} K_{\text {biloc }}^{-}=0$ due to the oscillating $(-1)^{k}$.

The previous analysis provides a new insight into the structure of fermionic entanglement: At any finite temperature, nonlocality couples a given point only to an infinite but discrete set of other points. The characteristic scale needed to resolve this discreteness goes as $1 / \beta$. Hence, continuous nonlocality emerges strictly in the limit of zero temperature. We summarize the structure of the modular Hamiltonian in Table I.

For multiple intervals, the only difference is that (22) now possesses one solution per interval for a given $k$, including the nontrivial $(x \neq y)$ solutions for $k=0$. In the low temperature limit, these extra terms yield precisely the bilocal terms of $[35,36]$.

During the final stage of this project, related results were independently reported in [43,44]. Equations (145) and (146) of [43] give the modular flow of the correlator. The generator of this flow corresponds to the expectation value of our result for the modular Hamiltonian. Finally, the versatility of the resolvent method has allowed us to compute the associated entanglement entropy [45], and can also be used to study other quantities related to the entanglement spectrum.

We are very grateful to D. Blanco and G. Pérez-Nadal for collaboration in the initial stages of this project. We thank J. Camps, B. Czech, M. Heller, H. Hinrichsen, C. Northe, and G. Wong for helpful discussions on the subject. P. F. is financially supported by the DFG Project No. DFG HI 744/9-1. The Gravity, Quantum Fields and Information group at AEI is generously supported by the Alexander von Humboldt Foundation and the Federal 
Ministry for Education and Research through the Sofja Kovalevskaja Award. I. R. also acknowledges the hospitality of Perimeter Institute, where part of this work was done.

*pascal.fries@physik.uni-wuerzburg.de †ignacio.reyes@aei.mpg.de

[1] S. A. Fulling, Nonuniqueness of canonical field quantization in Riemannian space-time, Phys. Rev. D 7, 2850 (1973).

[2] P. C. W. Davies, Scalar particle production in Schwarzschild and Rindler metrics, J. Phys. A 8, 609 (1975).

[3] W. G. Unruh, Notes on black hole evaporation, Phys. Rev. D 14, 870 (1976).

[4] S. W. Hawking, Euclidean quantum gravity, NATO Sci. Ser. B 44, 145 (1979).

[5] R. Haag, Local Quantum Physics: Fields, Particles, Algebras. Texts and Monographs in Physics (Springer, New York, 1992).

[6] H. Li and F. D. M. Haldane, Entanglement Spectrum as a Generalization of Entanglement Entropy: Identification of Topological Order in Non-Abelian Fractional Quantum Hall Effect States, Phys. Rev. Lett. 101, 010504 (2008).

[7] A. Chandran, M. Hermanns, N. Regnault, and B. A. Bernevig, Bulk-edge correspondence in entanglement spectra, Phys. Rev. B 84, 205136 (2011).

[8] M. Dalmonte, B. Vermersch, and P. Zoller, Quantum simulation and spectroscopy of entanglement Hamiltonians, Nat. Phys. 14, 827 (2018).

[9] I. Peschel and V. Eisler, Reduced density matrices and entanglement entropy in free lattice models, J. Phys. A 42, 504003 (2009).

[10] V. Eisler and I. Peschel, Properties of the entanglement Hamiltonian for finite free-fermion chains, J. Stat. Mech. (2018) 104001.

[11] W. Zhu, Z. Huang, and Y.-c. He, Reconstructing entanglement Hamiltonian via entanglement eigenstates, Phys. Rev. B 99, 235109 (2019).

[12] F. Parisen Toldin and F. F. Assaad, Entanglement Hamiltonian of Interacting Fermionic Models, Phys. Rev. Lett. 121, 200602 (2018).

[13] D. J. Luitz, N. Laflorencie, and F. Alet, Participation spectroscopy and entanglement Hamiltonian of quantum spin models, J. Stat. Mech. (2014) P08007.

[14] J. I. Cirac, D. Poilblanc, N. Schuch, and F. Verstraete, Entanglement spectrum and boundary theories with projected entangled-pair states, Phys. Rev. B 83, 245134 (2011).

[15] T. H. Hsieh, L. Fu, and X.-L. Qi, Tensor network implementation of bulk entanglement spectrum, Phys. Rev. B 90, 085137 (2014).

[16] L. Vanderstraeten, M. Mariën, J. Haegeman, N. Schuch, J. Vidal, and F. Verstraete, Bridging Perturbative Expansions with Tensor Networks, Phys. Rev. Lett. 119, 070401 (2017).

[17] G. Sárosi and T. Ugajin, Modular Hamiltonians of excited states, OPE blocks and emergent bulk fields, J. High Energy Phys. 01 (2018) 012.

[18] H. Casini, E. Teste, and G. Torroba, Modular Hamiltonians on the null plane and the Markov property of the vacuum state, J. Phys. A 50, 364001 (2017).
[19] H. Casini, Relative entropy and the Bekenstein bound, Classical Quantum Gravity 25, 205021 (2008).

[20] D. D. Blanco and H. Casini, Localization of Negative Energy and the Bekenstein Bound, Phys. Rev. Lett. 111, 221601 (2013).

[21] T. Faulkner, R. G. Leigh, O. Parrikar, and H. Wang, Modular Hamiltonians for deformed half-spaces and the averaged null energy condition, J. High Energy Phys. 09 (2016) 038.

[22] H. Casini, M. Huerta, and R. C. Myers, Towards a derivation of holographic entanglement entropy, J. High Energy Phys. 05 (2011) 036.

[23] D. D. Blanco, H. Casini, L.-Y. Hung, and R. C. Myers, Relative entropy and holography, J. High Energy Phys. 08 (2013) 060.

[24] D. L. Jafferis and S. J. Suh, The gravity duals of modular Hamiltonians, J. High Energy Phys. 09 (2016) 068.

[25] D. L. Jafferis, A. Lewkowycz, J. Maldacena, and S. J. Suh, Relative entropy equals bulk relative entropy, J. High Energy Phys. 06 (2016) 004.

[26] N. Lashkari, M. B. McDermott, and M. Van Raamsdonk, Gravitational dynamics from entanglement "thermodynamics”, J. High Energy Phys. 04 (2014) 195.

[27] J. Koeller, S. Leichenauer, A. Levine, and A. ShahbaziMoghaddam, Local modular Hamiltonians from the quantum null energy condition, Phys. Rev. D 97, 065011, 2018.

[28] Y. Chen, X. Dong, A. Lewkowycz, and X.-L. Qi, Modular flow as a disentangler, J. High Energy Phys. 12 (2018) 083.

[29] A. Belin, N. Iqbal, and S. F. Lokhande, Bulk entanglement entropy in perturbative excited states, SciPost Phys. 5, 024 (2018).

[30] R. Abt and J. Erdmenger, Properties of modular Hamiltonians on entanglement plateaux, J. High Energy Phys. 11 (2018) 002.

[31] R. Jefferson, Comments on black hole interiors and modular inclusions, SciPost Phys. 6, 042 (2019).

[32] J. J. Bisognano and E. H. Wichmann, On the duality condition for quantum fields, J. Math. Phys. (N.Y.) 17, 303 (1976).

[33] T. Hartman and N. Afkhami-Jeddi, Speed limits for entanglement, arXiv:1512.02695.

[34] J. Cardy and E. Tonni, Entanglement Hamiltonians in twodimensional conformal field theory, J. Stat. Mech. (2016) 123103

[35] H. Casini and M. Huerta, Reduced density matrix and internal dynamics for multicomponent regions, Classical Quantum Gravity 26, 185005 (2009).

[36] I. Klich, D. Vaman, and G. Wong, Entanglement Hamiltonians for Chiral Fermions with Zero Modes, Phys. Rev. Lett. 119, 120401 (2017).

[37] R. E. Arias, H. Casini, M. Huerta, and D. Pontello, Entropy and modular Hamiltonian for a free chiral scalar in two intervals, Phys. Rev. D 98, 125008 (2018).

[38] I. Klich, D. Vaman, and G. Wong, Entanglement Hamiltonians and entropy in $1+1 D$ chiral fermion systems, Phys. Rev. B 98, 035134 (2018).

[39] I. Peschel, Calculation of reduced density matrices from correlation functions, J. Phys. A 36, L205 (2003). 
[40] See Supplemental Material at http://link.aps.org/ supplemental/10.1103/PhysRevLett.123.211603 for details on some technical aspects of the calculation of both the resolvent and the modular Hamiltonian, including the computation of residues and the analysis of highly oscillatory distributions. We also specify our convention for the Jacobi theta functions and rewrite some of the expressions from the main text as a Lambert series.

[41] N. I. Muskhelishvili, Singular Integral Equations, 1st ed. (Springer, Netherlands, 1958).
[42] P. Di Francesco, P. Mathieu, and D. Senechal, Conformal Field Theory, Graduate Texts in Contemporary Physics (Springer-Verlag, New York, 1997), p. 457.

[43] S. Hollands, On the modular operator of mutli-component regions in chiral CFT, arXiv:1904.08201.

[44] D. Blanco and G. Prez-Nadal, Modular Hamiltonian of a chiral fermion on the torus, Phys. Rev. D 100, 025003 (2019).

[45] P. Fries and I. A. Reyes, companion paper, Eentanglement and relative entropy of a chiral fermion on the torus, Phys. Rev. D 100, 105015 (2019). 